\title{
Gain in Body Fat Is Associated with Increased Striatal Response to Palatable Food Cues, whereas Body Fat Stability Is Associated with Decreased Striatal Response
}

\author{
Eric Stice and Sonja Yokum \\ Oregon Research Institute, Eugene, Oregon, 97403
}

Cross-sectional brain-imaging studies reveal that obese versus lean humans show greater responsivity of reward and attention regions to palatable food cues, but lower responsivity of reward regions to palatable food receipt. However, these individual differences in responsivity may result from a period of overeating. We conducted a repeated-measures fMRI study to test whether healthy weight adolescent humans who gained body fat over a 2 or 3 year follow-up period show an increase in responsivity of reward and attention regions to a cue signaling impending milkshake receipt and a simultaneous decrease in responsivity of reward regions to milkshake receipt versus adolescents who showed stability of or loss of body fat. Adolescents who gained body fat, who largely remained in a healthy weight range, showed increases in activation in the putamen, mid-insula, Rolandic operculum, and precuneus to a cue signaling impending milkshake receipt versus those who showed stability of or loss of body fat, though these effects were partially driven by reductions in responsivity among the latter groups. Adolescents who gained body fat reported significantly greater milkshake wanting and milkshake pleasantness ratings at follow-up compared to those who lost body fat. Adolescents who gained body fat did not show a reduction in responsivity of reward regions to milkshake receipt or changes in responsivity to receipt and anticipated receipt of monetary reward. Data suggest that initiating a prolonged period of overeating may increase striatal responsivity to food cues, and that maintaining a balance between caloric intake and expenditure may reduce striatal, insular, and Rolandic operculum responsivity.

Key words: body fat gain; food cue; food receipt; incentive sensitization; long-term follow-up; repeated-measures fMRI

Significance Statement

This novel, repeated-measures brain-imaging study suggests that adolescents who gained body fat over our follow-up period experienced an increase in striatal responsivity to cues for palatable foods compared to those who showed stability of or loss of body fat. Results also imply that maintaining a balance between caloric intake and expenditure over time may reduce striatal, insular, and Rolandic operculum responsivity to food cues, which might decrease risk for future overeating.

\section{Introduction}

The incentive sensitization theory posits that repeated intake of high-calorie foods results in an elevated responsivity of reward and attention regions to cues repeatedly associated with hedonic reward from intake of such foods, and that this elevated responsivity prompts overeating when these cues are encountered (Berridge et al., 2010). Obese versus lean humans show greater

\footnotetext{
Received Dec. 5, 2015; revised May 18, 2016; accepted May 23, 2016.

Author contributions: E.S. and S.Y. designed research; S.Y. performed research; S.Y. analyzed data; E.S. and S.Y. wrote the paper.

This work was supported by National Institutes of Health Grant DK-080760. We thank the Lewis Center for Neuroimaging at the University of Oregon for their assistance with the fMRI scans.

The authors report no competing financial interests.

Correspondence should be addressed to Eric Stice, Oregon Research Institute, 1776 Millrace Drive, Eugene, OR 97403.E-mail: estice@ori.org.

DOI:10.1523/JNEUROSCI.4365-15.2016

Copyright $\odot 2016$ the authors $\quad 0270-6474 / 16 / 366949-08 \$ 15.00 / 0$
}

responsivity of brain regions implicated in reward [striatum, orbitofrontal cortex (OFC)] and attention (anterior cingulate cortex, precuneus) to pictures of high-calorie foods (Stoeckel et al., 2008; Martin et al., 2010; Stice et al., 2010a; Dimitropoulos et al., 2012). Intake of high-calorie foods versus tasteless foods results in greater activation of the OFC and greater striatal dopamine release, and the amount of dopamine release correlates positively with meal pleasantness ratings (O'Doherty et al., 2002; Small et al., 2003b). Anticipated receipt of a high-calorie food versus tasteless food results in greater activation in the OFC, amygdala, and striatum (O'Doherty et al., 2002; Pelchat et al., 2004; caudate nucleus and putamen). Elevated striatal and insula response to high-calorie food images correlates with higher appetitive ratings of the pictured food and greater subsequent ad libitum intake (Lawrence et al., 2012; Scharmüller et al., 2012). Animal experiments have found that frequent intake of high-calorie foods results in increased behavioral responsivity (e.g., licking cues), 
place preference, and resistance to extinction to cues that predict impending highcalorie food receipt (Flagel et al., 2009; Teegarden et al., 2009).

In contrast, the dynamic vulnerability theory posits that regular intake of high-calorie foods reduces reward region responsivity to tastes of such foods (Stice et al., 2011). Obese humans show less responsivity of striatal regions to tastes of high-calorie beverages than lean humans (Stice et al., 2008a; Green et al., 2011; Frank et al., 2012; Babbs et al., 2013). Animals randomized to overeating conditions that result in weight gain versus weight stable control conditions show a reduced sensitivity of reward circuitry to food intake, electrical stimulation, amphetamine administration, and potassium administration (Johnson and Kenny, 2010).

Although the cross-sectional data from

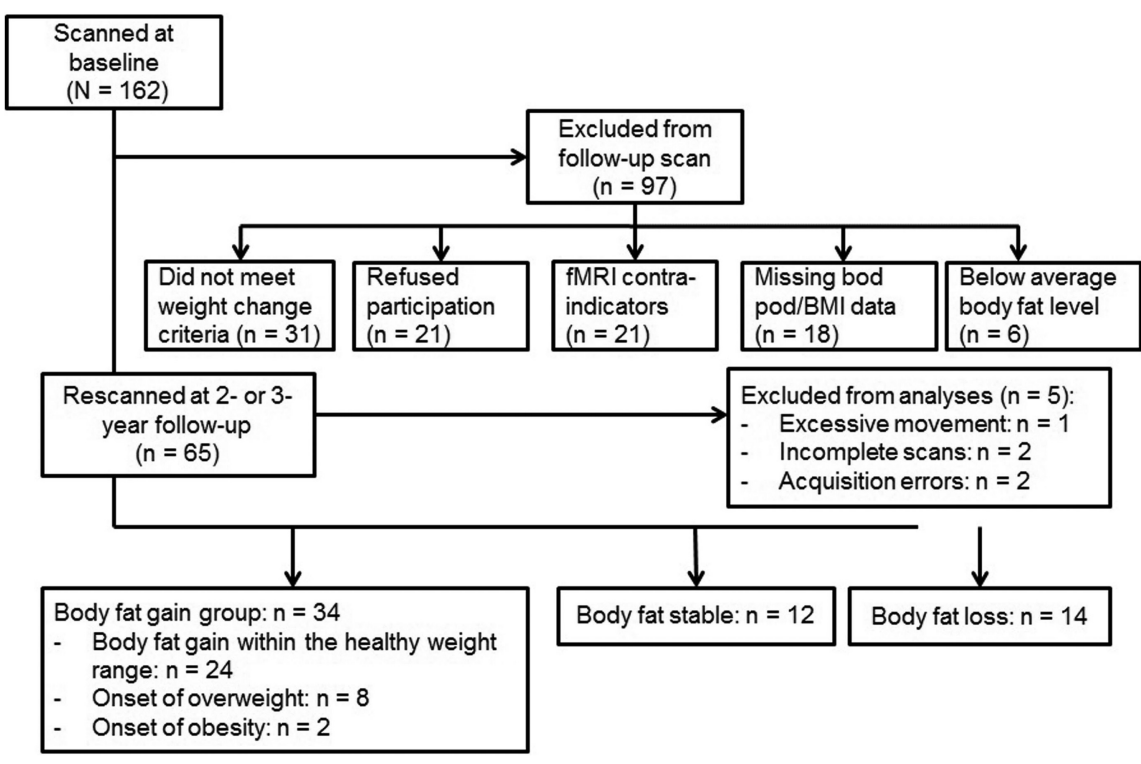
studies with humans appear consistent with the incentive sensitization and dynamic vulnerability theories of obesity, few repeated-measures studies with humans have tested these hypotheses. Humans randomly assigned to consume high-calorie foods daily over $2-3$ week periods show an increased willingness to work for that food and increased ad libitum intake of that food that is accompanied by a reduction in the reported "liking" of the foods relative to baseline and alternative highcalorie foods not consumed daily (Hetherington et al., 2000, 2002 Tey et al., 2012; Clark et al., 2010). However, no repeatedmeasures brain-imaging study with humans has tested whether chronic overeating that prompts initial excessive weight gain is associated with greater responsivity of reward and attention regions to cues for high-calorie foods. One repeated-measures study found that women who transitioned from overweight to obese over a 6 month follow-up showed a decrease in caudate responsivity to chocolate milkshake receipt relative to women who showed weight stability or loss over the follow-up period (Stice et al., 2010b). No study has tested whether weight gain among initially healthy weight individuals reduces reward region response to high-calorie foods. Herein, we test whether initially healthy weight adolescents who gained body fat over a longer 2 or 3 year follow-up period showed an increase in responsivity of reward and attention regions to a cue signaling impending chocolate milkshake receipt and a simultaneous decrease in responsivity of reward regions to chocolate milkshake receipt versus adolescents who showed body fat stability or loss over the follow-up period. Participants also completed a paradigm assessing neural response to the receipt and anticipated receipt of monetary reward to determine whether change in responsivity is specific to food receipt and cues.

\section{Materials and Methods}

\section{Participants}

One hundred and sixty-two healthy weight adolescents [ 82 female, 80 male; mean age, $15.3 \pm 1.1$; mean body mass index (BMI), $20.8 \pm 1.9$; mean body fat percentage, $18.4 \pm 7.7 ; 4 \%$ Hispanic, $1 \%$ Native American, $1 \%$ Asian/Pacific Islander, 76\% European American, 18\% mixed racial heritage] were recruited via advertisements for a 3 year prospective study. One hundred and twenty-five participants were at high risk for obesity [two obese or overweight $(B M I \geq 25)$ parents], and 37 were at low risk (two healthy weight parents). Exclusion criteria were a baseline

BMI (kilograms per square meter) of $<18$ or $>25$, current use of psychoactive medications or drugs more than weekly, pregnancy, head injury with a loss of consciousness, significant cognitive impairment, major medical problems, or a current Axis I psychiatric disorder. Adolescents and parents provided written informed consent for this institutional review board-approved project.

All participants completed the fMRI paradigms at baseline. Those who showed either $\mathrm{a} \geq 3 \%$ increase in body fat, $\mathrm{a} \geq 3 \%$ decrease in body fat, or less than a $2 \%$ change in body fat over a 2 or 3 year follow-up were invited to repeat the fMRI paradigms a second time at the 2 or 3 year follow-up. We operationalized body fat gain, loss, and stability in this fashion because based on the body fat change observed in the first year of follow-up, it seemed like these definitions would yield large enough cell sizes for adequate power. We scanned participants who satisfied the change in body fat criteria at the 2 year follow-up to maximize the sizes of the gain and loss groups, as we were concerned that they might not still satisfy the change criteria at the 3 year follow-up. However, if a participant showed further gain or loss in body fat, we invited them to repeat the scan at 3 year follow-up and used the data from that latter scan.

A participant flow chart is shown in Figure 1. Sixty-five participants (37\%) completed a second scan at the 2 or 3 year follow-up. Data from five participants were excluded from analyses due to excessive movement $(n=1)$, incomplete scans $(n=2)$, and acquisition errors $(n=2)$, resulting in $n=60$ (gain in body fat, $n=34$; stable body fat, $n=12$; loss of body fat, $n=14$ ). A total of 95 participants were not scanned due to (1) not meeting the change in body fat criteria (19\%), (2) declining the invitation to complete a follow-up scan (13\%), (3) reporting new fMRI contraindicators (e.g., braces) at follow-up (13\%), and (4) missing Bod Pod and BMI data at follow-up (12\%). We also decided not to scan six subjects ( $4 \%$; three males) who did show a $\geq 3 \%$ increase in body fat, but still remained below average body fat level $(7.7 \%$ body fat at follow-up for males, 7.7 or $16.8 \%$ body fat at follow-up for females).

\section{Measures}

Percent body fat. Air displacement plethysmography assessed body fat percentages of participants at baseline and at all follow-ups with the Bod Pod S/T (Life Measurement Inc./COSMED) using recommended procedures and age/sex appropriate equations (Lohman 1989). We focused on body fat rather than BMI because the former is a more precise measure of adipose tissue volume and is not influenced by variation in lean muscle mass. Body density was calculated as body mass (assessed by direct weighing) divided by body volume. Body fat percentage estimates show test-retest reliability $(r=0.92-0.99)$ and correlated with dual energy $\mathrm{x}$-ray absorptiometry and hydrostatic weighing estimates of body fat $(r=$ 
0.98-0.99; Fields et al., 2002). Nonetheless, we also assessed BMI so that we could fully characterize our sample. Height was measured to the nearest millimeter, and weight was assessed to the nearest $0.1 \mathrm{~kg}$ after removal of shoes and coats. Two measures of each were obtained and averaged.

Food and monetary reward fMRI paradigms. The food reward fMRI paradigm assessed responses to the receipt of palatable chocolate milkshake and tasteless solution $\left(25 \mathrm{~mm} \mathrm{KCl}\right.$ and $2.5 \mathrm{~mm} \mathrm{NaHCO}_{3}$ in distilled water) and to cues signaling the impending receipt of chocolate milkshake or tasteless solution. Images of glasses of milkshake and water signaled impending delivery of either $0.5 \mathrm{ml}$ of milkshake and tasteless solution, respectively. On $40 \%$ of the trials, the taste was not delivered following the cue to allow investigation of the neural response to anticipation of a taste that was not confounded with receipt of the taste (unpaired trials). However, because no difference in response was observed between paired and unpaired milkshake and tasteless solution cues, response to paired and unpaired cues were combined for analyses. In total, there were 30 repeats of both milkshake receipt and tasteless solution receipt and 50 repeats of both the milkshake cue and the tasteless solution cue that signaled a high probability of receipt of the beverages. On both scan days, participants were asked to consume their regular meals, but to refrain from eating or drinking caffeinated beverages for $5 \mathrm{~h}$ immediately preceding their scans. Participants rated pleasantness, wanting, and familiarity of the milkshake and tasteless solution and hunger before the scan using $20 \mathrm{~cm}$ cross-modal visual analog scales (VASs). VAS ratings were anchored by 0 (not at all), 10 (neutral), and 20 (never been more hungry). The monetary reward fMRI paradigm assessed activation in response to the receipt and anticipated receipt of monetary reward. First a coin on the left side of the screen alternated between blinking heads $(\mathrm{H})$ and tails (T) 2-4 times for $300 \mathrm{~ms}$ per blink and then "landed" on either $\mathrm{H}$ or T. After $2 \mathrm{~s}$, a second coin in the middle of the screen blinked 4-6 times before landing on $\mathrm{H}$ or T. After $3 \mathrm{~s}$, a third coin blinked 8-10 times on the right side of the screen before landing on $\mathrm{H}$ or $\mathrm{T}$. After the presentation of the three coins, a message appeared saying, "You win $\$ 3$ " or "You don't win." There were 20 win events (HHH or TTT displays), 30 win anticipation events (HH or TT displays), and 30 reward-neutral events (when a single $\mathrm{H}$ or $\mathrm{T}$ was displayed, which conveyed no information about the probability of winning). All participants won $\$ 48$ and received this amount once the scan was complete. Additional details regarding these paradigms have appeared previously (Stice et al., 2011).

Food and monetary reinforcement. Participants completed the progressive reinforcement paradigm developed by Epstein et al. (2003), wherein they worked to earn points toward a snack food reward of their choice and monetary reward to provide behavioral data on sensitivity to food and monetary reward. Participants first performed a taste test of $1 \mathrm{~g}$ of each food (M\&Ms, Reese's Peanut Butter Cups, Kit Kat, Cheetos, Pringles, Skittles, Oreo cookies, Gingersnaps, Gummy Bears, and popcorn), rating the pleasantness on visual analog scales and selecting the food they wanted to earn in the reinforcement task. In the second phase, three boxes varying in shape and color were displayed on a computer screen (similar to a slot machine). The boxes flipped, rotated, and changed color each time the participant pressed the mouse button. Points were earned each time the boxes matched in color and shape. The task started at a variable ratio (VR) $1 / 4$ schedule, meaning that, on average, one point was awarded for four button presses. The progressive VR schedule for the food item doubled (VR8, VR16, VR32, etc.) each time they earned five points. They were told that it would get progressively harder to earn points. The number of points earned for snacks was displayed at the top of the screen. Each five points earned was worth one standard serving of the food. Participants were told to play for as long as they liked. They then repeated this paradigm, but worked for $\$ 1$ monetary rewards, to provide a behavioral measure of sensitivity to an alternative reward. The breakpoint at which the participant stopped button pressing for food was used as the behavioral measure of food reward (i.e., how many button presses are made in total before the subject stops). A similar monetary reward breakpoint was calculated. The food reinforcement paradigm has shown 2-7 d test-retest reliability ( $r=0.80$; Epstein et al., 2007). Participants who rate the snack food as more pleasant work longer for it, participants work longer for snack food when calorically deprived, obese versus lean participants work longer for snack food, and participants who work longer for the snack consume more food ad libitum and show greater future weight gain (Epstein et al., 2003, 2007, 2014; Goldfield and Legg, 2006).

fMRI acquisition, preprocessing, and statistical analysis. A detailed description of the fMRI data acquisition is provided elsewhere, including main effects of these paradigms in terms of activation (Stice et al., 2011, 2012). Neuroimaging data were preprocessed and analyzed primarily using SPM12 (Functional Imaging Laboratory, University College London, London) in Matlab (MathWorks). Before preprocessing, all images were manually realigned to the anterior commissure-posterior commissure line in SPM and skull stripped using the Brain Extraction Tool in the FMRIB Software Library (FMRIB Analysis Group, Oxford, UK). During preprocessing in SPM, anatomical data were segmented and normalized using DARTEL, resulting in a sample-specific template and individuallevel deformation fields for application to the normalization step during functional data preprocessing. Functional data were (1) slice timing corrected, as these methods can successfully compensate for the temporal offset between slice acquisition and increase the robustness of the data analysis (Sladky et al., 2011); (2) adjusted for variation in magnetic field distortion using field maps (Poldrack et al., 2011); (3) realigned to the mean functional image from that run and coregistered with the anatomical image; and (4) normalized to MNI space using the DARTEL template and deformation fields output, which allows more precise alignment (Klein et al., 2009). Next, functional data were smoothed to $6 \mathrm{~mm}$ Gaussian FWHM. Functional data were then assessed to detect spikes in global mean response and motion outliers in the functional data using the Artifact Detection Toolbox (Gabrieli Lab, McGovern Institute for Brain Research, Cambridge, MA). Motion parameters were included as regressors in the design matrix at individual-level analysis. Additionally, image volumes where the $z$-normalized global brain activation exceeded 3 SDs from the mean of the run or showed $>1 \mathrm{~mm}$ of composite (linear plus rotational) movement were flagged as outliers and deweighted during individual-level model estimation.

To identify brain regions activated in response to chocolate milkshake receipt, we contrasted blood oxygen level-dependent (BOLD) activation during receipt of milkshake versus receipt of tasteless solution. To identify regions activated in response to the chocolate milkshake cue, we contrasted BOLD activation during presentation of the milkshake picture that signaled the impending delivery of the milkshake with response during presentation of the glass of water picture that signaled impending delivery of the tasteless solution. Activation in response to monetary reward was assessed by contrasting BOLD signal when participants saw the HHH or TTT displays versus the reward-neutral coin displays; activation in response to a cue signaling a potential monetary reward was assessed by contrasting BOLD activation when participants saw an $\mathrm{HH}$ or a TT display versus the reward-neutral coin displays.

At the individual level, T maps were constructed for comparison of activation within each participant for the four contrasts on the individual level (e.g., milkshake receipt $>$ tasteless solution receipt) at baseline and at follow-up. These individual contrasts were entered into repeatedmeasures ANOVA models to measure group differences (e.g., adolescents who gained body fat vs adolescents who showed stability of body fat) in change in neural response to the four contrasts over time (e.g., baseline chocolate milkshake receipt $>$ baseline tasteless solution receipt vs follow-up chocolate milkshake receipt $>$ follow-up tasteless solution receipt). Age at baseline and hunger levels before the scan (for the food reward fMRI paradigm analyses) were included as covariates. Wholebrain analyses were conducted after the binarized DARTEL-derived sample-specific gray matter mask was applied. An overall significance level of $p<0.05$ corrected for multiple comparisons across the gray matter-masked whole brain was calculated. This calculation was accomplished by (1) estimating the inherent smoothness of the masked functional data with the 3dFWHMx module in analysis of functional neuroimaging (AFNI) (Cox, 1996) and (2) performing 10,000 Monte Carlo simulations of random noise at $3 \mathrm{~mm}^{3}$ through the masked data using the 3DClustSim module of AFNI (Forman et al., 1995). Simulation results indicated activity surviving a threshold of $p<0.005$, with a cluster $(k) \geq 42$, is statistically significant, corrected for multiple comparisons. Effect sizes $(r)$ were derived from the $Z$ values $(Z / \sqrt{ } N)$. 
Table 1. Characterization of adolescents who gained body fat $(n=34)$, showed stable body fat $(n=12)$, or lost body fat $(n=14)$

\begin{tabular}{|c|c|c|c|c|c|}
\hline & Gain in body fat $(n=34)$ & Stable body fat $(n=12)$ & Loss of body fat $(n=14)$ & $F_{(2,57)}$ & $p$ \\
\hline Males & $44.1 \%$ & $58.3 \%$ & $28.6 \%$ & 1.16 & 0.32 \\
\hline Obesity risk & $76.5 \%$ & $58.3 \%$ & $92.9 \%$ & 2.20 & 0.12 \\
\hline \multicolumn{6}{|l|}{ Baseline (mean \pm SD) } \\
\hline Age & $15.3 \pm 1.1$ & $15.4 \pm 1.2$ & $14.9 \pm 0.5$ & 1.15 & 0.32 \\
\hline Parental education & $4.4 \pm 1.0$ & $4.3 \pm 1.0$ & $3.8 \pm 1.3$ & 1.78 & 0.18 \\
\hline Body fat percentage & $19.8 \pm 6.8$ & $17.4 \pm 9.2$ & $22.8 \pm 5.4$ & 1.88 & 0.16 \\
\hline Body mass index & $22.0 \pm 1.5$ & $20.6 \pm 1.9$ & $20.3 \pm 1.4$ & 2.74 & 0.07 \\
\hline Hunger & $7.1 \pm 4.1$ & $10.0 \pm 4.6$ & $6.8 \pm 4.3$ & 2.26 & 0.11 \\
\hline Pleasantness milkshake & $14.7 \pm 1.9$ & $15.3 \pm 2.1$ & $13.4 \pm 3.3$ & 2.40 & 0.10 \\
\hline Wanting milkshake & $13.9 \pm 2.4$ & $13.6 \pm 2.8$ & $12.8 \pm 2.8$ & 1.00 & 0.37 \\
\hline Familiarity milkshake & $16.0 \pm 3.0$ & $13.2 \pm 3.7$ & $15.0 \pm 4.0$ & 2.98 & 0.06 \\
\hline Food reinforcement & $2.3 \pm 0.5$ & $2.1 \pm 0.3$ & $2.4 \pm 0.6$ & 1.78 & 0.18 \\
\hline Monetary reinforcement & $2.9 \pm 0.7$ & $2.4 \pm 0.6$ & $2.8 \pm 0.6$ & 2.84 & 0.07 \\
\hline \multicolumn{6}{|l|}{ Follow-up (mean \pm SD) } \\
\hline Age & $17.8 \pm 1.4$ & $17.7 \pm 1.3$ & $17.1 \pm 0.7$ & 1.80 & 0.18 \\
\hline Follow-up scan (months from baseline) & $28.6 \pm 5.9$ & $27.0 \pm 5.4$ & $27.4 \pm 5.6$ & 0.43 & 0.66 \\
\hline Body fat percentage & $26.6 \pm 9.8$ & $17.6 \pm 9.5$ & $17.5 \pm 6.7$ & 7.23 & 0.00 \\
\hline Body mass index & $24.6 \pm 4.0$ & $21.5 \pm 2.1$ & $20.5 \pm 1.5$ & 9.23 & 0.00 \\
\hline Hunger & $10.1 \pm 3.8$ & $10.0 \pm 2.9$ & $11.1 \pm 3.7$ & 0.45 & 0.64 \\
\hline Pleasantness milkshake & $15.6 \pm 2.0$ & $15.4 \pm 1.2$ & $14.1 \pm 2.5$ & 3.07 & 0.04 \\
\hline Wanting milkshake & $15.6 \pm 2.5$ & $15.3 \pm 2.2$ & $12.6 \pm 2.8$ & 7.1 & 0.00 \\
\hline Familiarity milkshake & $16.6 \pm 3.9$ & $14.6 \pm 4.3$ & $16.3 \pm 3.4$ & 1.13 & 0.33 \\
\hline Food reinforcement & $2.1 \pm 0.4$ & $2.0 \pm 0.3$ & $2.1 \pm 0.4$ & 0.66 & 0.52 \\
\hline Monetary reinforcement & $2.4 \pm 0.5$ & $2.3 \pm 0.4$ & $2.4 \pm 0.4$ & 0.57 & 0.57 \\
\hline
\end{tabular}

\section{Results}

\section{Sample characteristics and behavioral data}

The groups showing differential change in body fat did not differ significantly on sex, parental overweight/obesity, or the following baseline variables: age; parental education; body fat percentage; BMI; hunger; pleasantness, wanting, and familiarity ratings of the milkshake taste; food reinforcement; and monetary reinforcement (Table 1). At follow-up, there were significant differences among the three groups in body fat percentage, BMI, and pleasantness and wanting ratings of the milkshake taste (Table 1). Pairwise contrasts showed that adolescents who gained body fat showed a higher body fat percentage compared to adolescents who showed stable body fat (effect size $d$ per Cohen, $1988=0.92$ ) or loss of body fat $(d=1.01)$. Adolescents who gained body fat also showed a higher BMI compared to adolescents who showed stable body fat $(d=0.97)$ or loss of body fat $(d=1.35)$. Adolescents who gained body fat reported higher milkshake pleasantness ratings compared to adolescents who lost body fat $(d=$ $0.60)$. Adolescents who gained body fat $(d=1.13)$ and those who showed stable body fat $(d=1.07)$ reported higher milkshake wanting ratings compared to adolescents who lost body fat. Paired $t$ tests were conducted to examine within-group changes in pleasantness, wanting, and familiarity ratings of the milkshake taste, food reinforcement, and monetary reinforcement. Bonferroni corrections were used to correct for the number of tests. Adolescents who gained body fat reported significant increases milkshake wanting ratings $\left(t_{(33)}=3.66, p<0.001\right)$, but did not show significant change in milkshake pleasantness ratings $\left(t_{(33)}=2.26\right.$, n.s.). Adolescents who gained body fat also reported significant decreases in the time they worked to earn snack food $\left(t_{(24)}=-3.18, p<0.01\right)$ and money $\left(t_{(24)}=-4.34, p<0.001\right)$. No significant within-group changes for these outcomes were observed in those who showed stable body fat and those who lost body fat.

At baseline, both wanting and pleasantness are positively associated with activation in the nucleus accumbens (wanting
MNI, 9, 3, $-6 ; Z=4.16, k=25$; pleasantness MNI, 9, 3, $-9 ; Z=$ $4.10, k=16)$ in response to milkshake receipt. Wanting and pleasantness were not significantly correlated with reward activation in response to anticipation of the milkshake receipt.

\section{Change in BOLD response to receipt and cues signaling impending receipt of milkshake between adolescents who gained body fat and those who showed stable body fat or loss of body fat}

Analyses comparing adolescents who gained body fat and those who showed stable body fat on change in BOLD activity in response to milkshake cue $>$ tasteless solution cue showed significant group by time interactions in the left putamen $(r=0.65)$, left mid-insula $(r=0.55)$, and right Rolandic operculum $(r=0.50$; Table 2). These interactions were partly driven by an increase in BOLD response for adolescents who gained body fat, in combination with a larger reduction in BOLD response in adolescents who showed stable body fat (Fig. $2 A-C$ ). Follow-up analyses explored whether within-group changes in BOLD activity were significant. Adolescents who gained body fat showed a significant increase in left putamen BOLD activation in response to milkshake cue $>$ tasteless solution cue $\left(t_{(33)}=2.76, p<0.01\right)$. The increases in BOLD activity in the left mid-insula and right Rolandic operculum were nonsignificant. Adolescents who showed stable body fat showed significant decreases in BOLD activity in the left putamen $\left(t_{(11)}=-4.10, p<0.01\right)$, left mid-insula $\left(t_{(11)}=\right.$ $-5.33, p<0.001)$, and right Rolandic operculum $\left(t_{(11)}=-5.04\right.$, $p<0.001)$ in response to milkshake cue $>$ tasteless solution cue. Follow-up analyses revealed that change in putamen BOLD activation did not correlate with change in milkshake pleasantness $(r=0.17)$, milkshake wanting $(r=-0.04)$, or food reinforcement $(r=-0.10)$. Changes in Rolandic operculum BOLD activation did not correlate with changes in milkshake pleasantness $(r=-0.11)$, milkshake wanting $(r=0.19)$, or food reinforcement $(r=0.06)$. Changes in insula BOLD activation did not correlate with change in milkshake pleasantness $(r=0.07)$, milk- 


\begin{tabular}{|c|c|c|c|c|}
\hline Contrasts and regions & $k$ & Z value & MNI coordinates & $r$ \\
\hline \multicolumn{5}{|l|}{ Gain > stable } \\
\hline \multicolumn{5}{|c|}{ Milkshake cue $>$ tasteless solution cue } \\
\hline Putamen & 51 & 4.38 & $-27,-15,0$ & 0.65 \\
\hline Mid-insula & 46 & 3.74 & $-39,3,3$ & 0.55 \\
\hline Rolandic operculum & 48 & 3.42 & $57,3,6$ & 0.50 \\
\hline \multicolumn{5}{|l|}{ Gain $>$ loss } \\
\hline \multicolumn{5}{|c|}{$\begin{array}{l}\text { Milkshake cue }>\text { tasteless solution cue } \\
\text { Follow-up }>\text { baseline }\end{array}$} \\
\hline Precuneus & 63 & 3.59 & $-12,-51,33$ & 0.52 \\
\hline Precuneus & & 3.29 & $-9,-42,48$ & 0.47 \\
\hline \multicolumn{5}{|l|}{ Milkshake $>$ tasteless solution } \\
\hline Superior parietal lobe & 45 & 4.72 & $-33,-63,51$ & 0.68 \\
\hline
\end{tabular}

For all contrasts, activated regions, $Z$ values, and coordinates within the MNI coordinate system are displayed. Numbers of contiguous voxels ( $k$ ) are shown for peak coordinates. All reported peaks were significant at $p<0.05$, corrected for multiple comparisons across the brain.

shake wanting ( $r=-0.16)$, or food reinforcement $(r=-0.09)$. There were no significant group by time differences in BOLD signal in response to milkshake receipt $>$ tasteless solution receipt.

Analyses comparing adolescents who gained body fat versus those who lost body fat on change in BOLD activity in response to milkshake cue $>$ tasteless solution cue showed significant group by time interactions in the left precuneus ( $r$ values $=0.52$ and 0.47 ; Table 2). These two interactions were primarily driven by reductions in BOLD response in adolescents who lost body fat (Fig. 2D). Follow-up analyses showed that the increase in precuneus activity in response to milkshake cue $>$ tasteless solution cue in adolescents who gained body fat was nonsignificant, but that the decrease in precuneus activity among adolescents who lost body fat in response to this same contrast was significant $\left(t_{(13)}=-2.49, p<0.05\right)$. Analyses comparing adolescents who gained body fat versus those who lost body fat on change in BOLD activity in response to milkshake receipt $>$ tasteless solution receipt showed significant group by time interactions in the left superior parietal lobe $(r=0.68)$. Adolescents who gained body fat showed a reduction in left superior parietal lobe activation from baseline to follow-up, and adolescents who lost body fat showed an increase in BOLD activity in this region (Fig. 2E). Follow-up analyses showed that adolescents who gained body fat showed a significant decrease in BOLD activity in the left superior parietal lobe $\left(t_{(33)}=-2.19, p<0.05\right)$ in response to milkshake receipt $>$ tasteless solution receipt, whereas adolescent who lost body fat showed a significant increase in left superior parietal lobe BOLD activity $\left(t_{(13)}=4.24, p<0.001\right)$ in response to this contrast. Changes in precuneus BOLD activation did not correlate with changes in milkshake pleasantness $(r=-0.04)$, milkshake wanting $(r=-0.01)$, and food reinforcement $(r=-0.01)$. Changes in superior parietal lobe BOLD activation did not correlate with changes in milkshake pleasantness $(r=0.06)$, milkshake wanting $(r=-0.06)$, or food reinforcement $(r=-0.11)$.

Change in BOLD response to receipt and anticipated receipt of monetary reward between adolescents who gained body fat and those who showed stable body fat or loss of body fat

There were no significant group by time differences in BOLD signal in response to potential monetary reward $>$ the rewardneutral coin display and in response to monetary reward $>$ the reward-neutral coin display across groups of adolescents who showed differential change in body fat.

\section{Discussion}

Adolescents who gained body fat exhibited an increase in activation in the putamen, mid-insula, and Rolandic operculum in response to the cue signaling impending milkshake receipt relative to adolescents who showed stable body fat. Research has found that the putamen, which in conjunction with the caudate nucleus forms the dorsal striatum, and the mid-insula respond to images of high-calorie foods and cue signaling impending palatable food receipt (Stice et al., 2012, 2013a; Tang et al., 2012). The Rolandic operculum, an oral somatosensory region, has also been found to respond to palatable food receipt (Stice et al., 2013a) and has been associated with reported reward from food intake (Small et al., 2003a). These findings extend previous findings of obese versus lean individuals showing greater activation in the striatum, insula, and Rolandic operculum during cues predicting impending palatable food receipt (Stice et al., 2008b) and in response to pictures of high-calorie foods versus low-calorie foods and control images (Stoeckel et al., 2008; Martin et al., 2010; Stice et al., 2010a; Dimitropoulos et al., 2012). The fMRI findings partially line up with behavioral evidence that adolescents who gained body fat reported greater milkshake wanting at follow-up than adolescents who lost body fat, though adolescents who showed stable body fat likewise reported greater milkshake wanting than adolescents who lost body fat. However, in contrast to expectations, the interactions involving BOLD response emerged in large part because the change in activation was reversed in adolescents who showed body fat stability versus body fat gain. The interactions shown in Figure $2 A-C$ indicate that adolescents who showed stable body fat exhibited greater reductions in activation in these regions than the corresponding increases among adolescents who gained body fat. Follow-up analyses showed that the increase in BOLD response among adolescents who showed body fat gain in response to milkshake cue $>$ tasteless solution cue was significant for the putamen, but not for the mid-insula or Rolandic operculum, whereas adolescents who showed stable body fat showed significant decreases in BOLD activity in all three regions (i.e., putamen, insula, and Rolandic operculum). This pattern of findings suggests that maintaining a healthy weight reduces food cue hyperresponsivity, which is a key neural vulnerability factor that predicts future weight gain (Demos et al., 2012; Yokum et al., 2011; Stice et al., 2015).

When comparing adolescents who gained body fat with those who lost body fat, we found a significant interaction in the precuneus in response to the cue signaling impending milkshake receipt. Follow-up within-condition analyses indicated that this interaction was mainly driven by the reduction in responsivity in the precuneus to food cues among adolescents who lost body fat. This finding converges with evidence that treatment-induced weight loss is associated with decreased responsivity in the precuneus to food cues (Pursey et al., 2014). The precuneus is a visual area that is functionally connected to reward regions (e.g., striatum and midbrain; $\mathrm{Ca}-$ vanna and Trible, 2006) and has been found to respond to cues predicting impending palatable food receipt (Stice et al., 2012). Adolescents who lost body fat also showed a greater increase in activation in the posterior superior parietal lobe to milkshake receipt than those who gained body fat. The posterior parietal lobe has been found to respond to palatable food receipt (Stice et al., 2011) and has been associated with attention to taste (Veldhuizen et al., 2012). 
A
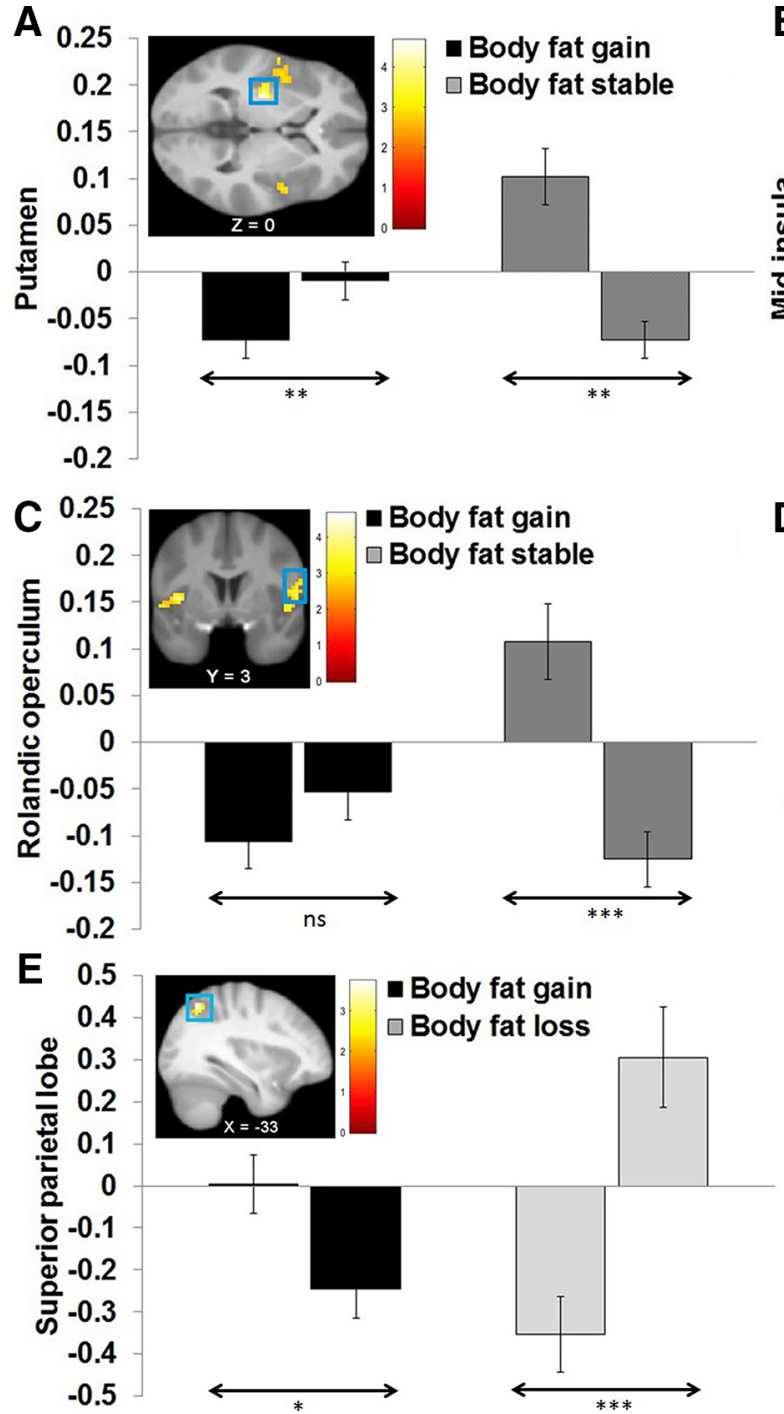

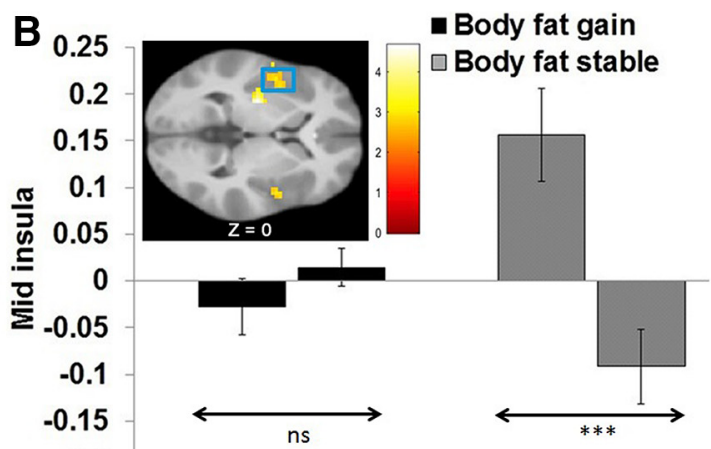

$-0.2$

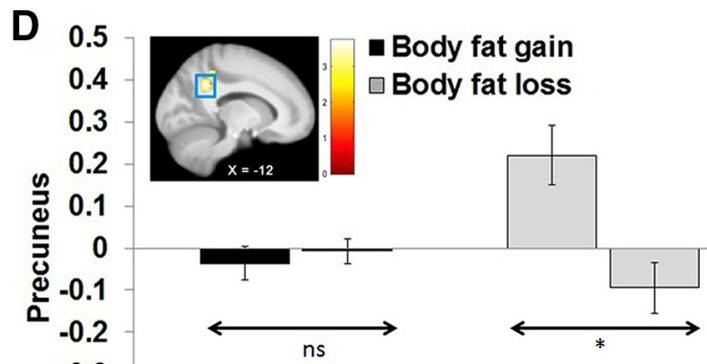

$-0.3$

$-0.4$

$-0.5$

Figure 2. $A-C$, Significant group by time interactions in left putamen (MNI, $-27,-15,0 ; Z=4.38, k=51 ; \boldsymbol{A})$, left mid-insula (MNI, $-39,3,3 ; Z=3.74, k=46 ; \boldsymbol{B})$, and right Rolandic operculum (MNI, 57, 3, 6; Z = 3.42, $K=48 ; C$ ) during milkshake cue/tasteless solution cue at follow-up compared with baseline in adolescents who showed gain versus stability in body fat. $\boldsymbol{D}, \boldsymbol{E}$, Significant group by time interactions in left precuneus ( $\mathrm{MNI},-12,-51,33 ; Z=3.59, k=63 ; \boldsymbol{D}$ ) during milkshake cue/tasteless solution cue and in left superior parietal lobe (MNI, -33 , $-63,51 ; Z=4.72, k=45 ; E$ ) during milkshake receipt/tasteless solution receipt in adolescents who showed gain versus loss in body fat. Double arrows below bar-plots represent within-condition changes. ${ }^{*} p<0.05 ;{ }^{* *} p<0.01 ;{ }^{* *} p<0.001$. ns, Not significant.

There was no evidence that healthy weight adolescents who gained body fat showed a reduction in striatal response to palatable food receipt relative to youth who showed stable body fat or loss of body fat. A previous study found that women who transitioned from being overweight to obese over a 6 month period showed a reduction in caudate response to milkshake receipt relative to women who showed a stable weight or weight loss over time (Stice et al., 2010b). The fact that most of the healthy weight adolescents who gained body fat in this study remained in a healthy weight or overweight range, rather than showing obesity onset, implies that downregulation of reward region response to palatable food may only occur with the transition to an obese state. In this context, it is important to note that the evidence that participants who gained body fat showed a significant decrease in how long they worked to earn snack foods seems inconsistent with the prediction from the dynamic vulnerability model that weight gain would lead to an increase in wanting of high-calorie foods (Stice et al., 2011). However, it is possible that the adolescents who gained the most weight were reluctant to exhibit a tendency to work for a very long time to earn snack foods because of self-presentation concerns. Results also indicated that adolescents who gained body fat rated the milkshake as more pleasant at follow-up compared to adolescent who lost body fat, though participants who gained body fat did not show a significant increase in milkshake pleasantness ratings over time.

Neural responses to the receipt of monetary reward and cues for monetary reward did not differ as a function of change in body fat, suggesting that gain in body fat changes neural responsivity that is specific to food reward, but not reward in general. The fact that we found multiple brain regions that showed altered activation after gain in body fat to receipt and cues signaling impending receipt of high-calorie food, but not to receipt and cues signaling impending receipt of monetary reward, also suggests that the former effects are reliable; a similar number of effects in response to both types of reward would be expected if results were due to chance. Yet, changes might have emerged for the food but not the monetary reward paradigm because the former provided a more sensitive measure of neural responsivity 
because the participants received the palatable food during the scans, but not the monetary reward. However, greater striatum response to monetary reward receipt in this paradigm did predict future substance use onset (Stice et al., 2013b), suggesting that this paradigm has predictive validity.

It is important to consider the study limitations. First, although the repeated-measures design increases sensitivity because each subject serves as their own control, and the fact that we observed numerous changes in BOLD response to palatable food receipt and cues that signal impending palatable food receipt confirmed that we had sufficient power to detect effects, the moderately small sample size limited sensitivity; this may explain why we did not observe changes in reward valuation regions, such as the caudate or orbitofrontal cortex. Second, we examined change in responsivity to only one pictorial cue of a milkshake. Future studies should test whether individuals who gain body fat show an increased responsivity of reward and attention regions to a broader range of palatable food images. Third, we only examined response to a single high-calorie palatable food; it is unclear whether results will generalize to receipt of other foods. Fourth, although we collected data regarding the pleasantness of the taste of milkshake, data on anticipated hedonic pleasure while viewing the cue that signaled impending delivery of the milkshake taste and attention allocation to that cue would have been useful in interpreting the meaning of the observed change in activation associated with body fat gain. Finally, when interpreting the potential meaning of activation in brain regions in our study, we sometimes used reverse inference, which is inferring certain cognitive processes based on the activation of a particular brain region (Poldrack, 2011). Although the intent is to extend our understanding of the function of particular brain regions, it is important to acknowledge this logic can be problematic because multiple cognitive processes can activate a particular brain region.

In sum, results suggest that adolescents who show body fat gain over a multiyear period experience an increase in striatal responsivity to cues for palatable food compared to adolescents who show body fat stability. The pattern of findings also implies that maintaining a balance between caloric intake and expenditure reduces striatal, insular, and Rolandic operculum responsivity to food cues, which may reduce risk for future overeating and weight gain.

\section{References}

Babbs RK, Sun X, Felsted J, Chouinard-Decorte F, Veldhuizen MG, Small D (2013) Decreased caudate response to milkshake is associated with higher body mass index and greater impulsivity. Physiol Behav 121: 103-111. CrossRef Medline

Berridge KC, Ho CY, Richard JM, DiFeliceantonio AG (2010) The tempted brain eats: pleasure and desire circuits in obesity and eating disorders. Brain Res 1350:43-64. CrossRef Medline

Cavanna AE, Trimble MR (2006) The precuneus: a review of its functional anatomy and behavioural correlates. Brain 129:564-583. CrossRef Medline

Clark EN, Dewey AM, Temple JL (2010) Effects of daily snack food intake on food reinforcement depend on body mass index and energy density. Am J Clin Nutr 91:300-308. CrossRef Medline

Cohen J (1988) Statistical power analysis for the behavioral sciences (2nd ed). Hillsdale, NJ; Lawrence Erlbaum.

Cox RW (1996) AFNI: software for analysis and visualization of functional magnetic resonance neuroimages. Comput Biomed Res 29:162-173. CrossRef Medline

Demos KE, Heatherton TF, Kelley WM (2012) Individual differences in nucleus accumbens activity to food and sexual images predict weight gain and sexual behavior. J Neurosci 32:5549-5552. CrossRef Medline

Dimitropoulos A, Tkach J, Ho A, Kennedy J (2012) Greater corticolimbic activation to high-calorie food cues after eating in obese vs. normalweight adults. Appetite 58:303-312. CrossRef Medline

Epstein LH, Truesdale R, Wojcik A, Paluch RA, Raynor HA (2003) Effects of deprivation on hedonics and reinforcing value of food. Physiol Behav 78:221-227. CrossRef Medline

Epstein L, Leddy J, Temple J, Faith M (2007) Food reinforcement and eating: a multilevel analysis. Psych Bull 133:884-906. CrossRef

Epstein LH, Yokum S, Feda DM, Stice E (2014) Parental obesity and food reinforcement predict future weight gain in non-obese adolescents. Appetite 82;138-142. CrossRef Medline

Fields DA, Goran MI, McCrory MA (2002) Body-composition assessment via air-displacement plethysmography in adults and children: a review. Am J Clin Nutr 75:453-467. Medline

Flagel SB, Akil H, Robinson TE (2009) Individual differences in the attribution of incentive salience to reward-related cues: implications for addiction. Neuropharmacology 56:139-148. CrossRef Medline

Forman BM, Tontonoz P, Chen J, Brun RP, Spiegelman BM, Evans RM (1995) 15-Deoxy-delta-12,14-prostaglandin $\mathrm{J}_{2}$ is a ligand for the adipocyte determination factor PPAR gamma. Cell 83:803-812. CrossRef Medline

Frank GK, Reynolds JR, Shott ME, Jappe L, Yang T, Tregellas JR, O’Reilly RC (2012) Anorexia nervosa and obesity are associated with opposite brain reward response. Neuropsychopharmacology 307:2031-2046. Medline

Goldfield G, Legg C (2006) Dietary restraint, anxiety, and the relative reinforcing value of snack food in non-obese women. Eat Behaviors 7: 323-332. CrossRef

Green E, Jacobson A, Haase L, Murphy C (2011) Reduced nucleus accumbens and caudate nucleus activation to a pleasant taste is associated with obesity in older adults. Brain Res 1386:109-117. CrossRef Medline

Hetherington M, Bell A, Rolls B (2000) Effects of repeated consumption on pleasantness, preference and intake. Brit Food J 102:507-521. CrossRef

Hetherington MM, Pirie LM, Nabb S (2002) Stimulus satiation: effects of repeated exposure to foods on pleasantness and intake. Appetite 38: 19-28. CrossRef Medline

Johnson PM, Kenny PJ (2010) Dopamine D2 receptors in addiction-like reward dysfunction and compulsive eating in obese rats. Nat Neurosci 13:635-641. CrossRef Medline

Klein SB, Cosmides L, Gangi CE, Jackson B, Tooby J, Costabile KA (2009) Evolution and episodic memory: an analysis and demonstration of a social function of episodic memory. Soc Cogn 27:283-319. CrossRef Medline

Lawrence NS, Hinto EC, Parkinson JA, Lawrence AD (2012) Nucleus accumbens response to food cues predicts subsequent snack consumption in women and increased body mass index in those with reduced selfcontrol. Neuroimage 63:415-422. CrossRef Medline

Lohman DF (1989) Human intelligence: an introduction to advances in theory and research. Rev Educ Res 59:333-374. CrossRef

Martin LE, Holsen LM, Chambers RJ, Bruce AS, Brooks WM, Zarcone JR, Butler MG, Savage CR (2010) Neural mechanisms associated with food motivation in obese and healthy weight adults. Obesity 18: 254-260. CrossRef Medline

O’Doherty JP, Deichmann R, Critchley HD, Dolan RJ (2002) Neural responses during anticipation of a primary taste reward. Neuron 33: 815-826. CrossRef Medline

Pelchat ML, Johnson A, Chan R, Valdez J, Ragland JD (2004) Images of desire: food-craving activation during fMRI. Neuroimage 23:1486-1493. CrossRef Medline

Poldrack RA (2011) Inferring mental states from neuroimaging data: from reverse inference to large-scale decoding. Neuron 72:692-697. CrossRef Medline

Poldrack RA, Kittur A, Kalar D, Miller E, Seppa C, Gil Y, Parker DS, Sabb FW, Bilder RM (2011) The cognitive atlas: towards a knowledge foundation for cognitive neuroscience. Front Neuroinform 5:17. Medline

Pursey KM, Stanwell P, Callister RJ, Brain K, Collins CE, Burrows TL (2014) Neural responses to visual food cues according to weight status: a systematic review of functional magnetic resonance imaging studies. Front Nutr 1:7. Medline

Scharmüller W, Übel S, Ebner F, Schienle A (2012) Appetitive regulation during food cue exposure: a comparison of normal-weight and obese women. Neurosci Lett 518:106-110. CrossRef Medline

Sladky R, Friston KJ, Tröstl J, Cunnington R, Moser E, Windischberger C 
(2011) Slice-timing effects and their correction in functional MRI. Neuroimage 58:588-594. CrossRef Medline

Small DM, Gregory MD, Mak YE, Gitelman D, Mesulam MM, Parrish T (2003a) Dissociation of neural representation of intensity and affective valuation in human gestation. Neuron 39:701-711. CrossRef Medline

Small D, Jones-Gotman M, Dagher A (2003b) Feeding-induced dopamine release in dorsal striatum correlates with meal pleasantness ratings in healthy human volunteers. Neuroimage 19:1709-1715. CrossRef Medline

Stice E, Spoor S, Bohon C, Small DM (2008a) Relation between obesity and blunted striatal response to food is moderated by TaqIA A1 allele. Science 322:449-452. CrossRef Medline

Stice E, Spoor S, Bohon C, Veldhuizen MG, Small DM (2008b) Relation of reward from food intake and anticipated intake to obesity: a functional magnetic resonance imaging study. J Abnorm Psychol 117:924-935. CrossRef Medline

Stice E, Yokum S, Bohon C, Marti N, Smolen A (2010a) Reward circuitry responsivity to food predicts future increases in body mass: moderating effects of DRD2 and DRD4. Neuroimage 50:1618-1625. CrossRef Medline

Stice E, Yokum S, Blum K, Bohon C (2010b) Weight gain is associated with reduced striatal response to palatable food. J Neurosci 30:13105-13109. CrossRef Medline

Stice E, Yokum S, Burger KS, Epstein LH, Small DM (2011) Youth at risk for obesity show greater activation of striatal and somatosensory regions to food. J Neurosci 31:4360-4366. CrossRef Medline

Stice E, Yokum S, Burger K, Epstein L, Smolen A (2012) Multilocus genetic composite reflecting dopamine signaling capacity predicts reward circuitry responsivity. J Neurosci 32:10093-10100. CrossRef Medline
Stice E, Burger KS, Yokum S (2013a) Relative ability of fat and sugar tastes to activate reward, gustatory and somatosensory regions. Am J Clin Nutr 98:1377-1384. CrossRef Medline

Stice E, Yokum S, Burger KS (2013b) Elevated reward region responsivity predicts future substance use onset but not overweight/obesity onset. Biol Psychiatry 73:869-876. CrossRef Medline

Stice E, Burger KS, Yokum S (2015) Reward region responsivity predicts future weight gain and moderating effects of the TaqIA allele. J Neurosci 35:10316-10324. CrossRef Medline

Stoeckel LE, Weller RE, Cook EW 3rd, Twieg DB, Knowlton RC, Cox JE (2008) Widespread reward-system activation in obese women in response to pictures of high-calorie foods. Neuroimage 41:636-647. CrossRef Medline

Tang DW, Fellows LK, Small DM, Dagher A (2012) Food and drug cues activate similar brain regions: a meta-analysis of functional MRI studies. Physiol Behav 106:317-324. CrossRef Medline

Teegarden SL, Scott AN, Bale TL (2009) Early life exposure to a high fat diet promotes long-term changes in dietary preferences and central reward signaling. Neuroscience 162:924-932. CrossRef Medline

Tey SL, Brown RC, Gray AR, Chisholm AW, Delahunty CM (2012) Longterm consumption of high energy-dense snack foods on sensory-specific satiety and intake. Am J Clin Nutr 95:1038-1047. CrossRef Medline

Veldhuizen MG, Gitelman DR, Small DM (2012) An fMRI study of the interactions between the attention and gustatory networks. Chemosens Percept 5:117-127. CrossRef Medline

Yokum S, Ng J, Stice E (2011) Attentional bias for food images associated with elevated weight and future weight gain: an fMRI study. Obesity 19:1775-1783. CrossRef Medline 